\title{
A randomised study on the clinical progress of high-risk elective major gastrointestinal surgery patients treated with olive oil-based parenteral nutrition with or without a fish oil supplement
}

\author{
María B. Badía-Tahull ${ }^{1}$, Josep M. Llop-Talaverón ${ }^{1}$, Elisabet Leiva-Badosa ${ }^{1}$, Sebastiano Biondo $^{2}$, \\ Leandre Farran-Teixidó ${ }^{2}$, Josep M. Ramón-Torrell ${ }^{3}$ and Ramón Jódar-Masanes ${ }^{1}$ \\ ${ }^{1}$ Pharmacy Service, Hospital Universitari de Bellvitge, IDIBELL, C/Feixa Llarga s/n, L'Hospitalet de Llobregat, \\ 08907 Barcelona, Spain \\ ${ }^{2}$ Digestive Surgery Service, Hospital Universitari de Bellvitge, IDIBELL, L'Hospitalet de Llobregat, Barcelona, Spain \\ ${ }^{3}$ Preventive Medicine Service, Hospital Universitari de Bellvitge, IDIBELL, L'Hospitalet de Llobregat, Barcelona, Spain
}

(Received 15 July 2009 - Revised 3 March 2010 - Accepted 5 March 2010 - First published online 30 March 2010)

n-3 Fatty acids have clinical benefits. The primary aim of the present study was the assessment of infection in patients who underwent major highrisk elective gastrointestinal surgery receiving postoperatively fish oil (FO)-supplemented parenteral nutrition (PN), compared with those receiving a standard olive oil (OO) emulsion. The secondary aims were the assessment of anti-inflammatory response and evaluation of tolerance and safety of these emulsions. A prospective, randomised, double-blind study was performed in patients requiring at least $5 \mathrm{~d}$ of PN. An isoenergetic and isoproteic formula was administered: group A received OO alone, while group B received OO that was partially replaced with FO (16.6\%, w/w). End points were outcome measures (mortality, sepsis, infection, hospitalisation days and PN duration), inflammatory response (C-reactive protein (CRP), prealbumin and leucocytes) and safety (TAG and glucose metabolism, and liver and kidney function). Statistical analysis was done using Student's $t$ test and Fisher's exact test $(P<0.05)$. Twenty-seven patients were evaluated, with thirteen patients receiving FO. In this group, a significantly lower incidence of infections was found $(23.1 \mathrm{v} .78 .6 \%, P=0.007)$. CRP, prealbumin and leucocytes were not significantly different between the groups. There were no differences in safety parameters. We conclude that high-risk surgical patients receiving FO-supplemented PN for $5 \mathrm{~d}$ present a lower incidence of infection. Emulsions were safe and well tolerated.

Total parenteral nutrition: Fish oil: Olive oil: Gastrointestinal surgery: Infection

Critically ill patients and those who have undergone surgery are at a high risk of developing infectious complications due to, among other reasons, a compromised immune status caused by the inflammatory process they experience ${ }^{(1)}$. Lipids in parenteral nutrition (PN) provide a major source of energy, essential fatty acids and liposoluble vitamins, as well as cell membrane components and eicosanoid and cytokine precursors, and they are attributed pharmacological functions that may intervene in the clinical course of the disease ${ }^{(2)}$.

The first soya oil-based lipid emulsions were associated with an altered response in inflammatory processes, which was suggested to contribute to greater susceptibility to infection and a poorer clinical outcome ${ }^{(3)}$. The concern over this potentially harmful consequence has led to the development of alternative lipid emulsions. Olive oil (OO)-based emulsions provide an immunoneutral option ${ }^{(3)}$ with clinical advantages $^{(3-5)}$ and proven tolerance and safety ${ }^{(4)}$. Fish oil (FO) emulsions are considered immunomodulators due to their capacity to modify both the synthesis of bioactive metabolites such as eicosanoids, released with hydrolysis of cell membrane phospholipids, and cytokine production ${ }^{(2)}$.

Despite these theoretical advances, there are few clinical trials examining the definitive impact of administering a single pharmaconutrient on the patients' clinical outcome ${ }^{(6,7)}$, and none of them directly compares OO and FO.

The primary aim of the present study was to assess the relationship between infection and FO-supplemented PN compared to a standard $\mathrm{OO}$ emulsion following a high-risk elective major surgery. The secondary aims included assessment of the patients' anti-inflammatory response and evaluation of the safety of, and tolerance to, these emulsions.

\section{Methods \\ Setting and study design}

A prospective, randomised, double-blind study was performed in a 900-bed, third-level teaching hospital in Barcelona

Abbreviations: FO, fish oil; OO, olive oil; PN, parenteral nutrition.

Conference presentation: This work was presented as a poster in the 30th Congress of ESPEN, Florence, Italy, 13-16 September 2008. It was selected as one of the 100 best communications of the Congress. Clinical trial registration number: 2008-007005-36.

* Corresponding author: María B. Badía-Tahull, fax + 34 932607507, email mbadia@bellvitgehospital.cat 
(Spain) in patients undergoing elective gastrointestinal tract major surgery. The sample size was calculated according to one hypothesis based on qualitative variables $(50 \%$ reduction in the incidence of infections) and another hypothesis based on quantitative variables $(25 \%$ difference in C-reactive protein values) by considering an $\alpha$ error of $5 \%$ and statistical power of $80 \%$. The final population required was therefore proposed to comprise at least twelve patients per group.

Patients were randomly allocated to two groups by consecutively assigning them a number from a randomisation table. Patients who were assigned an even number were placed in the control group, and those who were assigned an odd number were placed in the intervention group.

\section{Patients and inclusion criteria}

The study included adult patients who had undergone an elective gastrointestinal major surgery and required at least $5 \mathrm{~d}$ of PN. Patients were excluded for metabolic disease, chronic kidney or liver failure, severe haemorrhagic disorder, unstable diabetes, shock, sepsis, congestive heart disease, acute myocardial infarction, stroke, coma of unknown cause, HIV, dyslipoproteinaemia, neutropaenia, contraindications for lipid administration, current immunosuppressive or antineoplastic treatment, obesity or low weight, PN during the $15 \mathrm{~d}$ before hospitalisation, surgical intervention within $30 \mathrm{~d}$ before hospitalisation and expected fatal outcome.

\section{Study period}

From October 2002 to June 2003, twenty-nine patients were randomised. The study began once the patients had undergone surgery and the need for PN had been established. PN was started the day after the surgery. Patients were followed up from the day of the surgery until the day of discharge from the hospital. Seven different teams participated in the surgical interventions. Patients were hospitalised in the postoperative recovery unit, intensive care unit, and/or general surgery and gastrointestinal wards.

\section{Nutritional intervention}

PN was administered as 'all-in-one' mixtures that were prepared daily in the Pharmacy Service, and was delivered in masked containers. Neither the patient nor the expert assessor knew which lipid emulsion was being used. All PN formulas were administered using an infusion pump over $24 \mathrm{~h}$. Oral or enteral ingestion was not allowed.

Group A (control) received PN with OO lipid emulsion, and group $\mathrm{B}$ (intervention) received $\mathrm{PN}$ where a part of the $\mathrm{OO}$ emulsion had been substituted by FO in a proportion of $16.6 \%(\mathrm{w} / \mathrm{w})$, providing the same final amount of lipids.

Both groups received the same supply of nitrogen $(0 \cdot 2-0 \cdot 3 \mathrm{~g} / \mathrm{kg}$ per $\mathrm{d})$, energies $(83.7-125.5 \mathrm{~kJ} / \mathrm{kg}$ per $\mathrm{d}$ or $20-30 \mathrm{kcal} / \mathrm{kg}$ per $\mathrm{d})$ and lipids $(0.7-1 \mathrm{~g} / \mathrm{kg}$ per d). All patients received the same solution of amino acids and glucose (Aminomix ${ }^{\circledR}$; Fresenius-Kabi, Barcelona, Spain), vitamins (Cernevit ${ }^{\circledR}$; Baxter, Madrid, Spain) and trace elements (Addamel ${ }^{\circledR}$; Fresenius-Kabi). Commercial preparations were used for the administration of FO (Omegaven ${ }^{\circledR}$; FreseniusAB, Bad Homburg, Germany) and OO (Clinoleic ${ }^{\circledR}$; Baxter).
OO emulsions contain $20 \%$ soya oil. They are rich in long-chain MUFA, mainly $n-9$, and contain $20 \%$ PUFA with an $8: 1$ ratio of $n-6: n-3$. FO-based lipids, rich in $n-3$ PUFA, present a $1: 8$ ratio of $n-6: n-3$, and contain high amounts of $n$-3 family fatty acids (EPA and DHA) and vitamin E.

\section{Blood extraction}

Analytical parameters were determined on the day of the surgery, before starting $\mathrm{PN}$, on day 6 and after $5 \mathrm{~d}$ of PN administration. Blood was drawn at approximately 07.00 hours. Determinations were performed at the Clinical Laboratory of our hospital, which has been certified according to the UNE-EN-ISO 15189:2003 standard.

\section{Clinical follow-up}

Follow-up included daily monitoring of vital signs, allergic reactions, signs of infection, cardiac alterations, gastrointestinal tract dysfunction, lung processes, renal or hepatic dysfunction, haematological signs and behavioural disorders. Concomitant medication, fluid replacement therapy and blood administration were recorded.

\section{Clinical outcome}

The incidence of death, sepsis and non-septic infection was recorded, as well as both the number of hospitalisation days and the days PN treatment was received. Non-septic infection was established by clinical evidence and at least one positive culture from samples of lung (sputum or bronchoalveolar lavage), urine, blood, abdomen (peritoneal fluid or abdominal abscess) or surgical wound. Sepsis was defined on the basis of at least one positive culture plus two or more of the following conditions: leucocytes $>12 \times 10^{9}$ cells $/ 1$ or $<4 \times 10^{9}$ cells $/ \mathrm{l}$, body temperature $<35^{\circ} \mathrm{C}$ or $>38^{\circ} \mathrm{C}$, heart rate $>90 / \mathrm{min}$ and $\mathrm{PaCO}_{2}<32 \mathrm{mmHg}$.

\section{Inflammatory response}

The evolution of the inflammatory response was determined by C-reactive protein, prealbumin and leucocyte count after $5 \mathrm{~d}$ of PN.

\section{Safety of the infusion}

TAG and glucose metabolism, hepatic function ( $\gamma$-glutamyltransferase, alkaline phosphatase and alanine aminotransferase) and renal function (urea and creatinine) were specifically determined on day 6 following surgery to assess tolerance to the infused emulsion.

\section{Statistical analysis}

Student's $t$ test and Fisher's exact test were performed. Significance was set at a $P$ value $<0 \cdot 05$. SPSS 13.0 was used for the calculations (SPSS, Inc., Cary, NC, USA).

The present study was conducted according to the guidelines stated in the Declaration of Helsinki, and all procedures involving human patients were approved by the 'Comitè Ètic 
d'Investigació Clínica de l'Hospital Universitari de Bellvitge'. Written informed consent was obtained from all patients.

\section{Results}

\section{Clinical characteristics}

Initially, twenty-nine patients were randomised, but two patients were excluded. Among the twenty-seven patients assessed (median age 65 years; range $32-84$ ), 66.6\% ( $n$ 18) were men.

Twelve patients had large gastric adenocarcinoma (IIIA or IIIB). All the surgeries were open total gastrectomy with large lymphadenectomy; splenectomy was performed in six cases and pancreatectomy in four cases. In seven cases, a partial colectomy and partial small intestine resection were performed. Blood transfusion was necessary in ten cases. PN was indicated because the option of performing a successful jejunostomy for feeding was not considered an option.

Ten patients had pancreatic ductal adenocarcinoma (IIB or III). They underwent open duodenopancreatectomy, and all received blood transfusion, and in all but one, surgery took $6 \mathrm{~h}$ or more. After this kind of intervention, $\mathrm{PN}$ is the support diet regimen most frequently used in our hospital.

Three patients had oesophageal adenocarcinoma (IIB or III). They underwent open oesophagectomy (radical resection and reconstruction), and gastrectomy was also performed in two cases. They received PN because their nutritional status before the intervention was not considered adequate.

Two patients underwent colectomy, both with open surgery. One patient underwent a total colectomy with partial resection of small intestine because of a massive polyposis. The other patient underwent a combination of colectomy, hysterectomy, pancreatectomy and partial gastrectomy because of a gynaecological cancer with metastasis. Both patients received blood transfusion and finished with one or more stomata. The length of these interventions was over $6 \mathrm{~h}$. They received PN because of the extensive resections.
Fourteen patients received standard PN (group A), and thirteen patients received PN supplemented with FO (group B). The baseline characteristics of both groups are presented in Table 1. Use of concomitant medication and blood-related products showed no significant differences (data not shown). PN administration was carried out with no side effects in any case, and there were no reported problems in this regard. The nutrients administered are presented in Table 2.

\section{Outcomes}

Mortality, sepsis, infectious complications, hospital stay and PN duration are presented in Table 2. The only statistically significant difference was found in infectious complications: eleven in group A (three respiratory, three abdominal, four urinary and one surgical wound) and three in group B (one abdominal, one surgical wound and one bloodstream infection) $(P=0 \cdot 007)$.

\section{Inflammatory response and safety of the infusions}

No significant differences were found between the groups (Table 2).

\section{Discussion}

A significantly lower incidence of non-septic infection was observed in the FO-supplemented group.

FO acts as an immunomodulator ${ }^{(8-14)}$ with protective properties in the hyperinflammatory state of postoperative patients $^{(13)}$. The low content of $n-6$ and high- $\alpha$-tocopherol ${ }^{(15)}$ may contribute to an improvement in the oxidative state by minimising lipid peroxidation ${ }^{(13)}$. It must be emphasised that this outcome may be attributed to FO, but the influence of any particular component of this emulsion has not been studied in the present study.

Table 1. Baseline patient characteristics

(Mean values and standard deviations; $n$ and percentages)

\begin{tabular}{|c|c|c|c|c|c|c|c|}
\hline \multirow[b]{2}{*}{ Patients (n 27) } & \multicolumn{3}{|c|}{$\mathrm{OO}$} & \multicolumn{3}{|c|}{$\mathrm{OO}+\mathrm{FO}$} & \multirow[b]{2}{*}{$P$} \\
\hline & Mean & SD & $\%$ & Mean & SD & $\%$ & \\
\hline Age (years) & 63.71 & $14 \cdot 8$ & & 63.61 & $16 \cdot 24$ & & $0.87^{\star}$ \\
\hline \multicolumn{8}{|l|}{ Gender $(n)$} \\
\hline Men & 11 & & 78.5 & 7 & & $53 \cdot 8$ & $0.21 \dagger$ \\
\hline Women & 3 & & 21.4 & 6 & & $46 \cdot 2$ & \\
\hline $\mathrm{BMI}\left(\mathrm{kg} / \mathrm{m}^{2}\right)$ & 23.45 & 2.95 & & 23.21 & 4.92 & & $0.79^{*}$ \\
\hline Albumin $(g / l)$ & $27 \cdot 84$ & $7 \cdot 23$ & & 28.55 & 5.53 & & $0.54^{\star}$ \\
\hline C-reactive protein (mg/l) & $209 \cdot 80$ & 106 & & $172 \cdot 80$ & 48.90 & & $0.33^{*}$ \\
\hline Surgery (min) & 307 & 117 & & 275 & 107 & & $0.21^{*}$ \\
\hline Normal renal function $(n)$ & 13 & & 92.8 & 11 & & $84 \cdot 6$ & $0.56 \dagger$ \\
\hline Normal hepatic function $(n)$ & 10 & & 71.4 & 10 & & 73.9 & $0.78 \dagger$ \\
\hline \multicolumn{8}{|l|}{ Surgery $(n)$} \\
\hline Oesophagectomy & & 1 & & & 2 & & $0.23 \dagger$ \\
\hline Gastrectomy & & 6 & & & 6 & & $0.80 \dagger$ \\
\hline Duodenopancreatectomy & & 6 & & & 4 & & $0.44 \dagger$ \\
\hline Colectomy & & 1 & & & 1 & & $0.87 \dagger$ \\
\hline
\end{tabular}

OO, olive oil; FO, fish oil.

* Student's $t$ test.

$\dagger$ Fisher's exact test. 
Table 2. Parenteral intake, inflammatory response, emulsion safety and outcomes (Mean values and standard deviations; $n$ and percentages; medians and interquartile ranges (IQR))

\begin{tabular}{|c|c|c|c|c|c|}
\hline & \multicolumn{2}{|c|}{$\mathrm{OO}(n 14)$} & \multicolumn{2}{|c|}{$\mathrm{OO}+\mathrm{FO}(n 13)$} & \multirow[b]{2}{*}{$P^{*}$} \\
\hline & Mean & SD & Mean & SD & \\
\hline \multicolumn{6}{|l|}{ Nutritional facts } \\
\hline Nitrogen $(\mathrm{g} / \mathrm{kg}$ per $\mathrm{d}$ ) & 0.22 & 0.02 & 0.23 & 0.03 & 0.88 \\
\hline Glucose $(g / k g$ per $d)$ & $3 \cdot 15$ & 0.38 & 3.42 & 0.40 & 0.82 \\
\hline Lipid (g/kg per d) & 0.88 & $0 \cdot 10$ & 0.88 & 0.08 & 0.88 \\
\hline Volume $(\mathrm{ml} / \mathrm{d})$ & 2124 & 234 & 2201 & 255 & 0.73 \\
\hline Non-protein energy $(\mathrm{kJ} / \mathrm{kg}$ per $\mathrm{d})$ & $56 \cdot 5$ & $6 \cdot 7$ & $59 \cdot 2$ & 8.5 & 0.69 \\
\hline Total energy $(\mathrm{kJ} / \mathrm{kg}$ per $\mathrm{d})$ & $93 \cdot 1$ & 9.5 & 99.9 & $10 \cdot 8$ & 0.71 \\
\hline Lipid (\% non-protein energy) & 39.40 & 2.79 & $38 \cdot 31$ & $2 \cdot 78$ & 0.78 \\
\hline Glucose (\% non-protein energy) & $56 \cdot 67$ & $2 \cdot 75$ & $57 \cdot 69$ & $2 \cdot 78$ & 0.79 \\
\hline \multicolumn{6}{|l|}{ Analytical parameters } \\
\hline C-reactive protein (mg/l) & $188 \cdot 3$ & 94.3 & $142 \cdot 3$ & $69 \cdot 3$ & 0.16 \\
\hline Prealbumin (mg/l) & 83.0 & $53 \cdot 0$ & $105 \cdot 0$ & $37 \cdot 0$ & 0.22 \\
\hline Leucocytes $\left(\times 10^{9}\right.$ cells/l) & 13.68 & $9 \cdot 2$ & $9 \cdot 8$ & 3.9 & 0.17 \\
\hline TAG (mmol/l) & 1.77 & 0.82 & 1.81 & 0.49 & 0.89 \\
\hline Glucose $(\mathrm{mmol} / \mathrm{l})$ & $9 \cdot 28$ & 3.43 & $9 \cdot 27$ & 4.79 & 0.99 \\
\hline GGT $(\mu \mathrm{Kat} / \mathrm{l})$ & 3.81 & $3 \cdot 14$ & 3.94 & $2 \cdot 81$ & 0.91 \\
\hline $\operatorname{ALP}(\mu \mathrm{Kat} / \mathrm{l})$ & $2 \cdot 48$ & 0.66 & $2 \cdot 69$ & 1.46 & 0.65 \\
\hline ALT $(\mu \mathrm{Kat} / \mathrm{l})$ & 1.00 & 0.79 & 0.94 & 0.53 & 0.79 \\
\hline Urea (mmol/l) & $9 \cdot 32$ & 5.00 & $8 \cdot 23$ & $2 \cdot 00$ & 0.46 \\
\hline Creatinine $(\mu \mathrm{mol} / \mathrm{l})$ & $80 \cdot 7$ & $30 \cdot 10$ & $81 \cdot 62$ & $21 \cdot 30$ & 0.92 \\
\hline Outcomes & $n$ & $\%$ & $n$ & $\%$ & $P \dagger$ \\
\hline Mortality & 2 & $14 \cdot 3$ & 1 & $7 \cdot 7$ & 0.529 \\
\hline \multirow{3}{*}{ Infection } & 5 & $35 \cdot 7$ & 1 & $7 \cdot 7$ & 0.098 \\
\hline & 11 & $78 \cdot 6$ & 3 & $23 \cdot 1$ & 0.007 \\
\hline & Median & IQR & Median & IQR & $P^{*}$ \\
\hline Hospital stay (d) & 15 & 12 & 16 & 17 & 0.544 \\
\hline Duration of PN (d) & 8 & 4.5 & 7 & 3.75 & 0.442 \\
\hline
\end{tabular}

OO, olive oil; FO, fish oil; GGT, $\gamma$-glutamyltransferase; ALP, alkaline phosphatase; ALT, alanine aminotransferase; $\mathrm{PN}$, parenteral nutrition.

* Student's $t$ test.

† Fisher's exact test.

Results of related studies are in agreement with the fact that the infection rate is lower in groups that receive FO, but the differences found have not been shown to be statistically significant ${ }^{(11,16)}$. Indeed, the overall incidence of non-septic infection in the present study is high, which could be attributed to the fact that the patients who were selected were seriously ill, and had undergone quite complicated major surgery; nevertheless, no significant difference was observed for sepsis between the groups.

Differences regarding mortality were NS, which was consistent with other studies ${ }^{(2,6,17,18)}$. The high rate of mortality observed in the present study could be attributed to the facts that all the diseases were malignant in a high stage and were treated through open surgery and in long interventions, and that the albumin values before the intervention were low.

Clearly, there are two main limitations to the present study: the small sample size and the lack of more inflammatory and immunological markers. However, it should be noted that the main focus of the study was the patients' clinical outcome.

This is the first study that has compared the addition of FO to an emulsion based on $\mathrm{OO}$ that has proven metabolic efficacy and safety ${ }^{(19)}$ and, in some studies, immunoneutral properties $^{(20)}$. It has been hypothesised that FO emulsions may have an associated immune suppression which could reduce the host resistance to infection ${ }^{(5)}$. The present results provide statistical support to the idea that the protective activity of these lipids and the high amounts of vitamin $\mathrm{E}$ predominate over an immunosuppressive effect with possible enhanced susceptibility to infection. The maximum dose of FO is established at $0 \cdot 2 \mathrm{~g} / \mathrm{kg}$ per $\mathrm{d}^{(1,7)}$, although the proper balance between $n-3$ and $n-6$ remains uncertain. In the present study, the ratio was $1: 1 \cdot 6$, with a content of $0 \cdot 13-0 \cdot 16 \mathrm{~g}$ of $\mathrm{FO} / \mathrm{kg}$ per $\mathrm{d}$.

In conclusion, this is the first PN study that attempted to define the impact of FO supplementation $v$. an OO-based emulsion. The strength of the study in a limited number of high-risk surgical patients during $5 \mathrm{~d}$ of PN was not sufficient to assess the overall impact of $\mathrm{OO} v$. FO supplementation. Nonetheless, the FO-supplemented regimen demonstrated a clear and statistically significant reduction in non-septic infection. Further studies in larger patient populations with more extensive investigation of specific inflammatory markers are needed to reassess these findings.

\section{Acknowledgements}

The authors would like to thank the staff of the Departments of Gastrointestinal Surgery and Pharmacy, and Celine Cavallo and Jonathan Rogerson for the translation. There are no 
conflicts of interest. The translation was funded by IDIBELL. M. B. B.-T. carried out the studies and data analyses and drafted the manuscript; J. M. L.-T. conceived the study, carried out the statistical analysis and corrected the manuscript; E. L.-B. participated in the design of the study and corrected the manuscript; S. B.-A. and L. F.-T. participated in the design and coordination of the study; J. M. R.-T. and R. J.-M. revised the manuscript. All authors read and approved the final manuscript.

\section{References}

1. Heller AR, Fischer S, Rössel T, et al. (2002) Impact of $n$-3 fatty acid supplemented parenteral nutrition on haemostasis patterns alter major abdominal surgery. Br $J$ Nutr 87, Suppl. 1, S95-S101.

2. Tsekos E, Reuter C, Stehle P, et al. (2004) Perioperative administration of parenteral fish oil supplements in a routine clinical setting improves patient outcome alter major abdominal surgery. Clin Nutr 23, 325-330.

3. Cury-Boaventura MF, Gorjao R, Martins de Lima $\mathrm{T}$, et al. (2008) Effect of olive oil-based emulsion on human lymphocyte and neutrophil death. JPEN 32, 81-87.

4. Goulet O, Potter S, Antébi H, et al. (1999) Long-term efficacy and safety of a new olive-oil based intravenous fat emulsion in pediatric patients: a double-blind randomized study. Am J Clin Nutr 70, 337-345.

5. Puertollano MA, Puertollano E, Álvarez de Cienfuegos G, et al. (2007) Significance of olive oil in the host immune resistance to infections. Br J Nutr 98, S54-S58.

6. Heller AR, Rössler S, Lits RJ, et al. (2006) Omega-3 fatty acids improve the diagnosis-related clinical outcome. Crit Care Med 34, 972-979.

7. Heller AR, Rösel T, Gottschlich B, et al. (2004) Omega-3 fatty acids improve liver and pancreas function in postoperative cancer patients. Int J Cancer 111, 611-616.

8. Waitzberg DL, Torrinhas RS \& Jacintho TM (2006) New parenteral lipid emulsions for clinical use. JPEN 30, 351-367.

9. Mayer K, Meyer S, Reinholz-Muhly M, et al. (2003) Short-time infusion of fish oil-based lipid emulsions, approved for parenteral nutrition, reduces monocyte proinflammatory cytokine generation and adhesive interaction with endothelium in humans. J Immunol 171, 4387-4843.

10. Mayer K, Fegbeutel C, Hattar K, et al. (2003) Omega-3 vs. omega-6 lipid emulsions exert differential influence on neutrophils in septic shock patients: impact on plasma fatty acids and lipid mediator generation. Intensive Care Med 29, 1472-1481.

11. Wanten GJ \& Calder PC (2007) Immune modulation by parenteral lipid emulsions. Am J Clin Nutr 85, 1171-1184.

12. Moussa M, Le Boucher J, Garcia J, et al. (2000) In vivo effects of olive oil-based lipid emulsion on lymphocyte activation in rats. Clin Nutr 19, 49-54.

13. Schlotzer E \& Kanning U (2004) Elimination and tolerance of a new parenteral lipid emulsion (SMOF) - a double-blind crossover study in healthy male volunteers. Ann Nutr Metab $\mathbf{4 8}$, 263-268

14. Mertes N, Grimm H, Fürst P, et al. (2006) Safety and efficacy of a new parenteral lipid emulsion (SMOFlipid) in surgical patients: a randomized, double-blind, multicenter study. Ann Nutr Metab 50, 253-259.

15. Antébi H, Mansoor O, Ferrier C, et al. (2004) Liver function and plasma antioxidant status in intensive care unit patients requiring total parenteral nutrition: comparison of 2 fat emulsions. JPEN 28, 142-148.

16. Senkal M, Geiger B, Hannemann M, et al. (2007) Supplementation of omega-3 fatty acids in parenteral nutrition beneficially alters phospholipids fatty acid pattern. JPEN 31, 12-17.

17. Grecu I, Mirea L \& Grintescu I (2003) Parenteral fish oil supplementation in patients with abdominal sepsis (abstract). Clin Nutr 22, S23.

18. Wang $\mathrm{X}, \mathrm{Li} \mathrm{W}, \mathrm{Li} \mathrm{N}$, et al. (2008) Omega-3 fatty acidssupplemented parenteral nutrition decreases hyperinflammatory response and attenuates systemic disease sequelae in severe acute pancreatitis: a randomized and controlled study. JPEN 32, 236-241.

19. García-de-Lorenzo A, Denia R, Atlan P, et al. (2005) Parenteral nutrition providing a restricted amount of linoleic acid in severely burned patients: a randomised double-blind study of an olive oil-based lipid emulsion v. medium/long-chain triacylglycerols. Br J Nutr 94, 221-230.

20. Grimm H, Mertes N, Goeters C, et al. (2006) Improved fatty acid and leukotriene pattern with a novel lipid emulsion in surgical patients. Eur J Nutr 45, 55-60. 